\title{
Guía para la valoración médico-forense de la rodilla
}

\section{Guideline for evaluation of the knee in forensic medicine}

\section{Introducción}

Aunque en principio parece que la exploración de la rodilla sea una más, similar a la de cualquier otra región del organismo, en realidad existen ciertas razones que justifican que se le preste una consideración especial. La patología de la rodilla es bastante frecuente en la práctica forense para peritar lesiones traumáticas de diversa etiología, ya sea por accidentes de tráfico, accidentes laborales, agresiones, caídas accidentales, lesiones deportivas, etc., muchas veces complementadas o superpuestas a complejas y variadas patologías previas. Es preciso conocer el mecanismo de producción de este tipo de lesiones para establecer con el mayor grado de certeza posible la relación de causalidad, debiendo descartar enfermedades preexistentes que deben catalogarse como estado anterior patológico (y/o concausa preexistente), y detectar lesiones postraumáticas residuales para valorarlas como secuelas, casi constantes en este tipo de lesiones.

Por otra parte, la referida patología es muy variable y específica, derivada de una complicada anatomía, que condiciona una biomecánica y una cinemática también muy complejas, a veces difícil de interpretar y que requiere precisos conocimientos y gran concreción para hacer una valoración médico-forense correcta.

Todo ello implica la práctica de unas correctas y minuciosas anamnesis y exploración, que abarca una serie de medidas generales y otras específicas de esta articulación, que pretendemos describir, para finalmente apuntar los ejes sobres los que debe descansar una correcta valoración de las secuelas.

\section{Exploración médico-forense de la rodilla}

\section{Anamnesis}

Debe ser cuidadosa, empezando por el mecanismo de producción de las lesiones, imprescindible para determinar la causalidad de estas, y para ello utilizaremos cuanta documentación se nos facilite (informes médicos, atestados, historiales clínicos previos, etc.). Es muy importante analizar las características del dolor, teniendo en cuenta que nunca irradia ni se expande más allá de la rodilla por las lesiones de esta, a diferencia de otras articulaciones, aunque en cambio puede haber dolor referido a este nivel procedente de la cadera, la columna lumbar o el tobillo. Hay que descartar asimismo el dolor producido por patologías linfáticas o vasculares no ortopédicas.

La anamnesis debe dirigirse a conocer el modo en que cumplen su función los miembros inferiores, descrito minuciosamente por Melennec: función de enderezamiento (tránsito desde la posición sedente hasta la ortostática, sin o con ayuda de las manos; bipedestación; marcha (en suelo llano, en pendiente y en suelo accidentado), duración de esta, necesidad de descanso, y calzado; ascenso y descenso de escaleras; adopción de posturas en cuclillas y de rodillas, y paso de la posición de rodillas a la ortostática con o sin ayuda de las manos.

\section{Exploración física general (Tabla 1)}

\section{Inspección}

Permite ver anomalías en la alineación de los miembros inferiores y detectar si hay desviaciones en varo, valgo o recurvatum (medición de la distancia intermaleolar mínima en valgo y de la intercondílea en varo). Asimismo, pueden observarse atrofias musculares de cuádriceps o vasto interno (medición comparativa de los perímetros de ambos muslos a $15-20 \mathrm{~cm}$ de la base de la rótula); marcas de la piel o cicatrices de intervenciones o exploraciones previas; inflamación articular o periarticular global o tumefacciones localizadas, tales como quistes poplíteos o de Baker, higromas, bursitis prerrotulianas o subrotulianas, o quistes meniscales, hidrartrosis o hemartrosis que deberán precisarse con la palpación.

\section{Á. Hernández Gil ${ }^{1}$ \\ J. Hernández Moreno² \\ L. Luna Alcalá ${ }^{3}$}

${ }^{1}$ Doctor en Medicina. Jefe de Servicio Clínica Médico Forense. Instituto de Medicina Legal. Jaén 2Doctor en Medicina. Médico Forense. Especialista en Traumatología y Medicina Legal y Forense. Jaén

${ }^{3}$ Especialista en Radiología. Coordinador Médico Territorial Grupo Health Time. Jaén.

Correspondencia: Ángel Hernández Gil Instituto de Medicina Legal de Jaén C/ San Antonio 5-7

23071 Jaén

E-mail: angel.hernandez.ius@ juntadeandalucia.es

Fecha de recepción: 12. JUN. 2014

Fecha de aceptación: 23. JUN. 2014 
Tabla 1. Exploración física general en la valoración médico-forense de la rodilla.
Anamnesis

Exploración física general

A. Inspección

Anomalías en la alineación

Atrofias musculares

Marcas en la piel o cicatrices

Tumefacciones localizadas (quistes, higromas)

Desviaciones en la situación de la rótula

Hidrartrosis o hemartrosis

Inflamación articular

Control de la marcha

B. Palpación

Calor local

Bloqueos y pseudobloqueos

Puntos dolorosos selectivos (regiones parameniscales y LLI LLE)

Crepitación y signos rotulianos: cepillo, test de Zöhlen

Test de estabilidad rotuliana

Palpación de huesos

Palpación de tejidos blandos

C. Movilidad Valorar en decúbito supino: flexión máxima $\left(135^{\circ}\right)$ y extensión $\left(0^{\circ}\right)$

Valorar rodilla flexionada $90^{\circ}$ : rotación externa $\left(25^{\circ}\right)$ y rotación interna $\left(10^{\circ}\right)$

Exploración específica ligamentosa y meniscal

Exploración complementaria
Revela también posibles desviaciones en la situación de la rótula hacia arriba, abajo y laterales, que deben completarse con la palpación y la movilización. Debe controlarse la marcha, y si hay claudicación o fallos en la misma.

\section{Palpación}

Permite apreciar:

- Aumento de calor local, que apunta a procesos inflamatorios o infecciosos.

- Derrame articular: hidrartrosis y hemartrosis traumáticas. La hidrartrosis aparece lentamente y produce molestias por distensión, en general sin calor local, y es propia de traumatismos de la membrana sinovial. La hemartrosis es de aparición rápida, y causa dolor muy agudo y calor local. Es consecuencia de la lesión de un vaso sanguíneo de la sinovial articular, debida a una lesión de los ligamentos cruzados o a una desinserción meniscal periférica, o bien a fracturas osteocondrales de las superficies óseas de la rodilla por arrancamiento de las espinas tibiales.

- Bloqueos y pseudobloqueos: el bloqueo es la aparición súbita de un chasquido doloroso y la imposibilidad de realizar la flexoextensión de la rodilla, quedando limitada en $25-40^{\circ}$ de flexión, pues el bloqueo siempre es incompleto y permite cierta movilidad con resistencia elástica. Ocurre cuando un fragmento meniscal roto queda atrapado entre el cóndilo femoral y la tibia, típicamente en las roturas en asa de cubo. El pseudobloqueo es un bloqueo momentáneo fácilmente reducible, que se produce en roturas pedunculadas del menisco interno.

- Puntos dolorosos: deben explorarse los puntos dolorosos selectivos de las regiones parameniscales interna y externa, así como las inserciones y el trayecto de los ligamentos laterales, que indicarán la región lesionada.

- Crepitación y signos rotulianos: signo del cepiIlo, que consiste en mover la rótula en sentido proximal y distal, y de forma medial y lateral presionándola contra los cóndilos femorales. La existencia de dolor nos indicará condromalacia rotuliana o artrosis femoropatelar. Test de Zöhlen o de la resistencia sostenida. Test de estabilidad rotuliana.

- Palpación de huesos: rótula, meseta tibial medial y lateral interna, tuberosidad tibial, tubérculo del aductor, cóndilos y epicóndilos femorales. 
- Palpación de tejidos blandos: en la región anterior pueden palparse el cuádriceps, el tendón rotuliano y las bolsas prerrotulianas y de la pata de ganso; en la región medial, el menisco interno, el ligamento lateral interno (LLI), la inserción de los tendones del sartorio, el vasto interno y el semitendinoso; en la región lateral, el menisco externo, el ligamento lateral externo (LLE), el tendón del bíceps crural, la cintilla iliotibial y el nervio ciático poplíteo externo; en la superficie posterior, la fosa poplítea, el nervio tibial posterior y los vasos poplíteos, y la inserción de los gemelos. Debe hacerse con la rodilla en flexión, pues en extensión algunos de los contornos de la rodilla desaparecen.

\section{Movilidad}

La flexoextensión debe valorarse en decúbito supino. La máxima flexión posible es de $135^{\circ}$ y la extensión es de $0^{\circ}$. Las rotaciones sólo son posibles con la rodilla en flexión. Con la rodilla colgando y en flexión de $90^{\circ}$, la rotación externa es de $25^{\circ}$ y la interna es de $10^{\circ}$. Debemos considerar que la rotación no se contempla en el Baremo anexo al RDL 8/2004.

\section{Exploración específica ligamentosa y meniscal (Tabla 2)}

\section{Signos meniscales}

Unos van destinados a provocar dolor y se conocen como signos funcionales; son más positivos en las fases agudas y en general están basados en movilizaciones múltiples en flexoextensión combinadas con rotaciones (Steinman I) o con varo o valgo (Böhler), o el desplazamiento del dolor a la presión en la interlínea articular hacia delante y atrás en lesiones del menisco interno (Steinman II). Similar es el signo de Bragard (en rotación interna y extensión de la rodilla, se provoca dolor en la parte anterior de la interlínea interna en caso de lesión del menisco interno).

\section{Anamnesis}

Exploración física general

Exploración específica ligamentosa y meniscal

Signos meniscales:

Funcionales (provocación de dolor):

Steinman I (flexoextensión + rotaciones interna y externa)

Bohler (flexoextensión + varo o valgo)

Steinman II (desplazamiento dolor interlínea hacia delante o atrás)

Bragard (dolor anterior en interlínea en extensión y rotación interna)

Mecánicos (atrapamiento segmento meniscal entre interlinea y cóndilo):

McMurray

Apley (decúbito prono con rodilla en flexión $90^{\circ}$ : presión y tracción)

Ligamentos laterales:

provocar el "bostezo articular"

Forzar el varo o el valgo (para LLE y LLI, respectivamente)

Maniobra de Moragas para LLE

Ligamentos cruzados:

Signos directos (evaluación de la integridad de los ligamentos):

Cajones anterior y posterior (neutro y rotaciones interna y externa)

Test de Latchman Trillat (cadera en extensión y rodilla a $15^{\circ}-20^{\circ}$ )

Pruebas dinámicas (valoración combinada de inestabilidad anteroposterior y externa):

Pivot shift de Mclntosh

Jerk test de Houston

Exploración complementaria 
Figura 1. Test de McMurray. Exploración de los meniscos. Para explorar el menisco interno se efectúa una flexión máxima de la cadera con el pie rotado externamente $(A)$ y una extensión gradual de la rodilla hasta los $90^{\circ}(B)$ mientras se mantiene la rotación externa, apareciendo chasquidos o dolor en el compartimento interno cuando está

lesionado. La exploración del menisco externo se realiza de la misma forma pero con la tibia en rotación interna y aducción.

Figura 2.

Stress en valgo.

Exploración de la integridad del LLI.

Una prueba positiva indica lesión de los LLI y posterior.

Si la articulación se abre ampliamente, es probable que estén también lesionados

los ligamentos cruzados.

Figura 3.
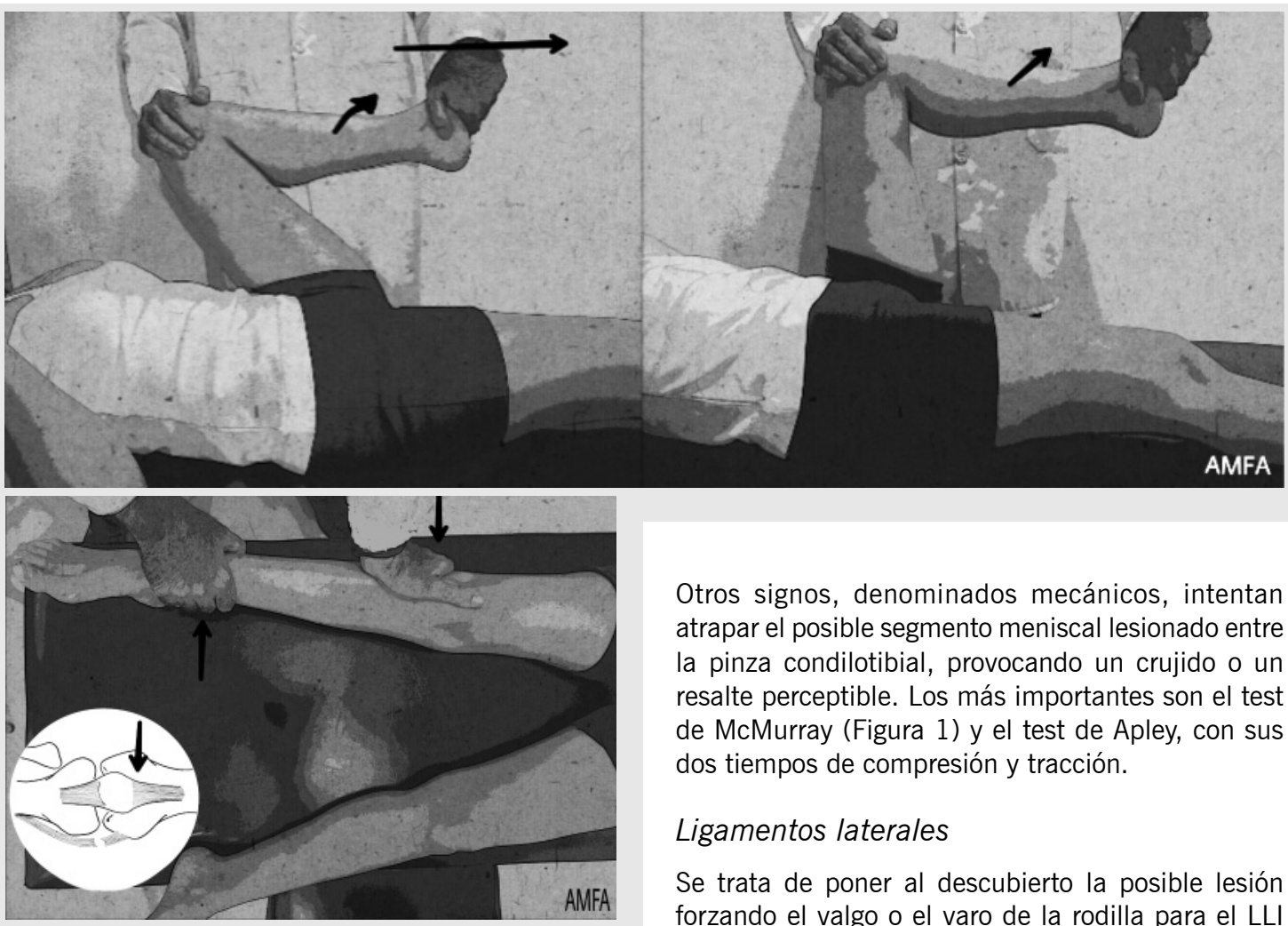

Stress en varo.

Exploración de la integridad del LLE.

Con una mano como punto de apoyo sobre el lado interno, se trata de abrir la articulación sobre el lado externo moviendo medialmente el pie.

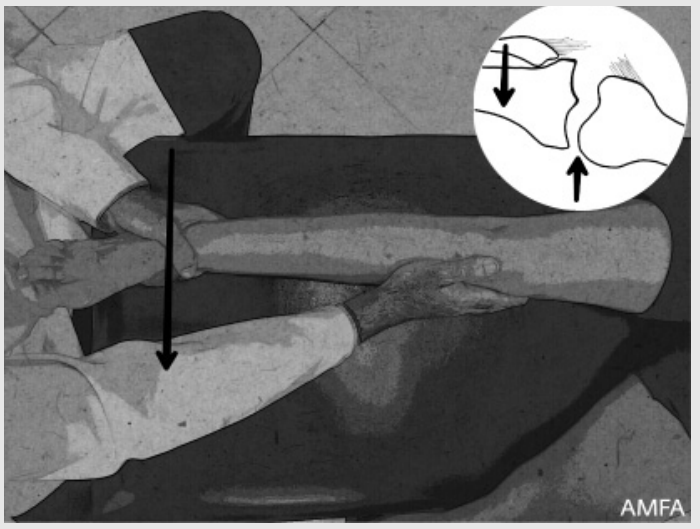

Figura 4.

Cajón anterior y posterior. Exploración de los LCA y LCP. Es conveniente comparar el grado de desplazamiento de

la tibia con el de la rodilla contraria. Un desplazamiento de $2 \mathrm{~cm}$ o más indica un desgarro en el ligamento cruzado anterior y posterior.

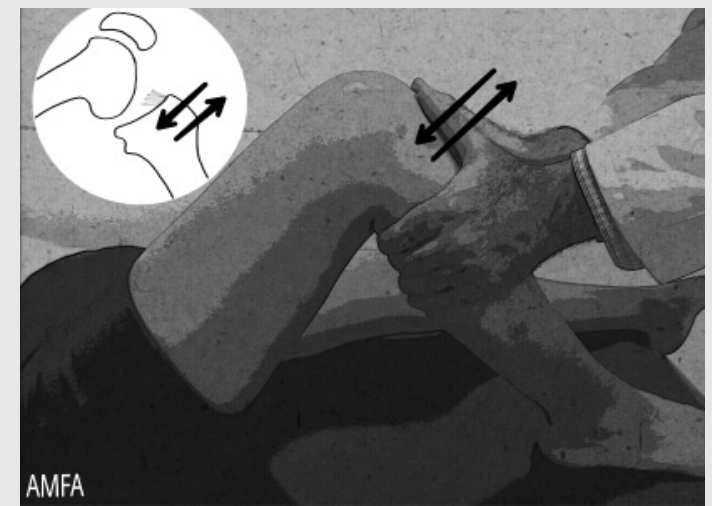

Otros signos, denominados mecánicos, intentan atrapar el posible segmento meniscal lesionado entre la pinza condilotibial, provocando un crujido o un resalte perceptible. Los más importantes son el test de McMurray (Figura 1) y el test de Apley, con sus dos tiempos de compresión y tracción.

\section{Ligamentos laterales}

Se trata de poner al descubierto la posible lesión forzando el valgo o el varo de la rodilla para el LLI (Figura 2) o el LLE (Figura 3), respectivamente, con el objetivo de apreciar el grado de inestabilidad lateral o interna, y de observar la presencia o no de bostezo articular, considerando la prueba positiva si lo hubiera. Para entreabrir la interlínea externa en las lesiones del LLE es muy simple y efectiva la maniobra de Moragas, que consiste en colocar la pierna en rotación externa y flexión, y el pie cruzado por encima de la pierna sana, y se palpa la alteración de la tensión normal del ligamento a ese nivel en caso de lesión.

\section{Ligamentos cruzados anterior (LCA) y posterior ( $(L C P)$}

Hay signos directos para evaluar la integridad de los ligamentos, los conocidos como "cajones anterior y posterior" (test de Slocum, Figura 4), que se practican con el sujeto en decúbito supino, con la cadera en flexión de $45^{\circ}$ y la rodilla en $90^{\circ}$, las plantas de los pies sobre la camilla y el explorador sentado junto al pie para sujetarlo. Se colocan las manos alrededor de la rodilla con los pulgares sobre la línea articular medial y lateral, y los índices en las inserciones medial y lateral de los músculos femorales posteriores, y se tira de la tibia hacia delante y hacia atrás, respectivamente, lo que origina un desplazamiento hacia delante (rotura del LCA) o hacia atrás (rotura del LCP), con la consiguiente deformación visible y palpable de la 
rodilla. La prueba debe hacerse primero en rotación neutra, pero después en rotación interna y externa, que son los llamados "cajones anteriores rotatorios interno y externo". Igualmente deben explorarse los "cajones combinados valgo y varo", que ponen de manifiesto las frecuentes asociaciones de la lesión de estos ligamentos con la de los LLI y LLE. En el test de Lachman-Trillat (anterior y posterior), los cajones se exploran con una flexión mínima (de unos $15^{\circ}$ ) de la rodilla, es decir, con la rodilla casi en extensión, sosteniendo con una mano el fémur y con la otra la tibia proximal, a la cual se le realiza desplazamiento anterior sosteniendo fijamente el fémur. Es el test más sensible para las lesiones de los ligamentos cruzados, pero es más difícil de realizar y comúnmente se hace en quirófano.

Las pruebas dinámicas permiten valorar la inestabilidad combinada anteroposterior y externa, y se basan en provocar o reducir la subluxación anterior de la meseta externa cuando la rodilla está cerca de la extensión. La más típica es el test de pivot shift o prueba del desplazamiento lateral del pivote de
McIntosh, que es una maniobra en la cual se realiza una flexoextensión de la rodilla aplicando a la vez una fuerza en valgo y en rotación interna. En los pacientes con lesión del LCA se observa cómo la rodilla se subluxa en extensión y se reduce en flexión. Esta prueba puede explorarse en sentido contrario en el denominado jerk test o prueba de la contracción súbita de Houston.

\section{Exploraciones complementarias (Tabla 3)}

Utilizadas casi de modo constante en el ámbito asistencial, su resultado dota de objetividad a los hallazgos apreciados en la exploración física, y en muchas ocasiones sirven para revelar lesiones que no han podido catalogarse en la exploración. Tales medios complementarios son la radiología, la tomografía computarizada (TC), la ecografía, la resonancia magnética (RM), la artroscopia y la medicina nuclear. A continuación se revisan someramente sus indicaciones principales, posibilidades, ventajas e inconvenientes.

\begin{tabular}{|c|c|c|c|}
\hline $\begin{array}{l}\text { Pruebas } \\
\text { complementarias }\end{array}$ & Ventajas & Inconvenientes & Indicaciones \\
\hline Radiología & $\begin{array}{l}\text { Fácil de realizar e interpretar } \\
\text { Idónea para visualizar } \\
\text { huesos y líneas articulares }\end{array}$ & $\begin{array}{l}\text { Superposición de tejidos } \\
\text { No visualiza elementos } \\
\text { articulares no radioopacos }\end{array}$ & $\begin{array}{l}\text { Lesiones óseas } \\
\text { Lesiones de ligamentos } \\
\text { laterales internos y externos }\end{array}$ \\
\hline Ecografía & $\begin{array}{l}\text { Rápida } \\
\text { Inocua } \\
\text { Accesible } \\
\text { Barata }\end{array}$ & $\begin{array}{l}\text { Difícil interpretación } \\
\text { por facultativos no } \\
\text { especializados }\end{array}$ & $\begin{array}{l}\text { Patología ligamentosa, } \\
\text { muscular, superficial } \\
\text { o periarticular }\end{array}$ \\
\hline $\begin{array}{l}\text { Tomografía } \\
\text { computarizada }\end{array}$ & $\begin{array}{l}\text { Concreta lesiones en } \\
\text { estructuras óseas o radioopacas }\end{array}$ & $\begin{array}{l}\text { Radiación excesiva } \\
\text { Menor disponibilidad } \\
\text { Mayor coste económico }\end{array}$ & $\begin{array}{l}\text { Precisar fracturas articulares } \\
\text { Valoración de estructuras } \\
\text { de densidad extraña }\end{array}$ \\
\hline $\begin{array}{l}\text { Resonancia } \\
\text { magnética }\end{array}$ & $\begin{array}{l}\text { Excelente visualización APGca } \\
\text { Ausencia de radiación } \\
\text { Revela patología no apreciada } \\
\text { en la exploración física }\end{array}$ & $\begin{array}{l}\text { Mayor tiempo de realización } \\
\text { Mayor coste económico } \\
\text { Difícil interpretación por } \\
\text { facultativos no especializados }\end{array}$ & $\begin{array}{l}\text { Patología meniscal, } \\
\text { ligamentosa y osteocondral } \\
\text { Tumores, infecciones y } \\
\text { necrosis } \\
\text { Fracturas ocultas }\end{array}$ \\
\hline $\begin{array}{l}\text { Medicina nuclear: } \\
\text { Gammagrafía, } \\
\text { Tomografía por } \\
\text { emisión de } \\
\text { positrones }\end{array}$ & $\begin{array}{l}\text { Seguras, sin riesgos } \\
\text { No dolorosas } \\
\text { Buena relación coste-beneficio } \\
\text { Imagen funcional del } \\
\text { metabolismo óseo }\end{array}$ & $\begin{array}{l}\text { Su gran sensibilidad para } \\
\text { muchas patologías } \\
\text { les resta especificidad }\end{array}$ & $\begin{array}{l}\text { Fracturas ocultas } \\
\text { o diagnóstico precoz } \\
\text { Entesopatías } \\
\text { Tumores, infecciones } \\
\text { Prótesis dolorosas }\end{array}$ \\
\hline
\end{tabular}

Tabla 3.

Pruebas complementarias en la valoración médico-forense de la rodilla. 
La radiología es el procedimiento de imagen empleado generalmente como primera elección, y es muy útil para la visualización de los elementos óseos, con la limitación que supone la superposición en un mismo plano de todas las estructuras atravesadas por los rayos $X$. Deben hacerse proyecciones anteroposterior y lateral, generalmente con flexión de $30^{\circ}$ y axial de la rótula cuando se sospeche patología de esta, y proyecciones especiales oblicuas o laterales, proyecciones forzadas de varo o valgo, proyección de Rosemberg y anteroposterior en $45^{\circ}$, para valorar mejor la interlínea.

La TC está indicada para completar el estudio de fracturas, valorar estructuras calcificadas o de densidad extraña (cuerpos libres articulares o lesiones blásticas), y como guía para punciones diagnósticas. Su utilización está influenciada por diversos factores: disponibilidad, resolución de imagen, coste-efectividad y radiación.

La ecografía es un método diagnóstico barato, rápido, accesible e inocuo (no requiere el uso de radiaciones ionizantes). Sus indicaciones en la exploración de la rodilla son el estudio de la patología muscular, tendinosa y ligamentaria superficiales, las lesiones quísticas periarticulares, la valoración del derrame articular, las masas superficiales y la patología vascular.

La RM es la prueba no invasiva de elección para valorar las lesiones internas de la rodilla. Se emplea para estudiar y valorar las lesiones de los meniscos, de los ligamentos y del cartílago articular, y es el método de elección en las lesiones osteocondrales. Se utiliza asimismo en el estudio de infecciones, tumores, necrosis y fracturas ocultas. Es una técnica no invasiva que facilita una excelente visualización anatomopatológica. Sin embargo, entre sus inconvenientes pueden citarse que requiere cierto tiempo para su realización y la posible falta de disponibilidad de ella, aunque de todos modos puede concluirse que tiene buena relación coste efectividad (Figuras 5-7).

Aunque escapan de las posibilidades médicoforenses, cabe mencionar otras pruebas complementarias, como la artroscopia, inicialmente con fines diagnósticos, pero en la actualidad casi siempre lleva asociada la terapéutica; la gammagrafía ósea, especialmente útil para diagnosticar artritis sépticas; y la tomografía por emisión de positrones, indicada en procesos oncológicos.

\section{Valoración médico-forense de las secuelas}

Con independencia de la causa de la lesión (agresión, accidente laboral, etc.), en la práctica, la principal herramienta de trabajo en la valoración de secuelas en el ámbito médico-forense es el Baremo anexo al RDL 8/2004 que indemniza las lesiones derivadas de accidentes de tráfico, si bien, a juicio de los autores,

Figura 5. Secuencia $T 2$ y DP Sag: rotura completa de ligamento cruzado anterior (LCA).
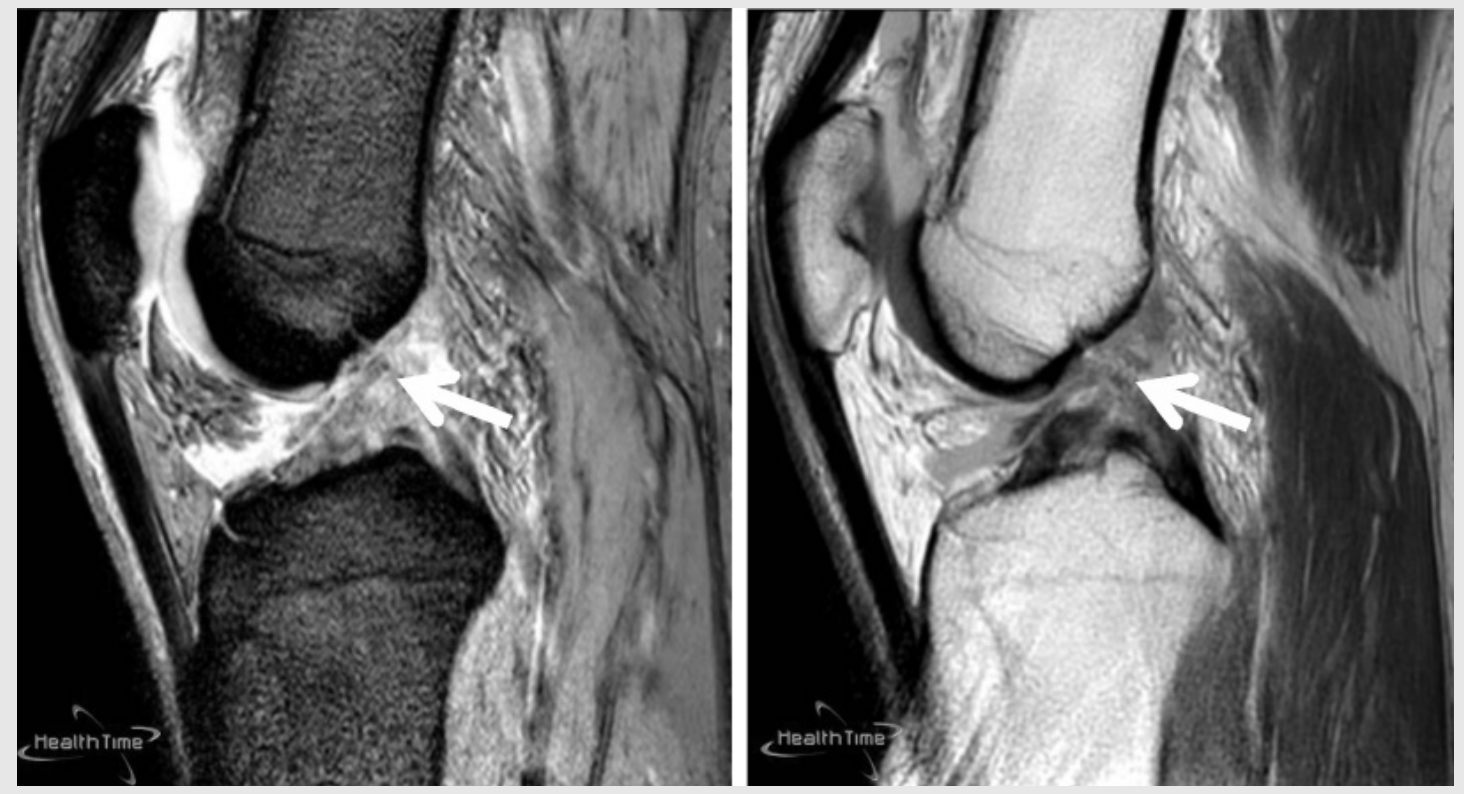
presenta algunas lagunas e insuficiencias, entre las que pueden citarse:

- Considera lesiones en vez de secuelas (discapacidades), de modo especial en las patologías más frecuentes de la rodilla en el ámbito médicoforense, que son las lesiones de los ligamentos laterales y cruzados y la patología meniscal, en la valoración de las cuales sólo se basa en la lesión en sí, en la práctica quirúrgica o no para el tratamiento, y en que quede o no sintomatología, resolviéndolo con abanicos inadmisibles (de 1 a 15 puntos para los cruzados, 1 a 10 puntos para los laterales y 1 a 5 puntos para los meniscos).

- Establece una secuela, la "artrosis postraumática", en la que incluye limitaciones funcionales y dolor, como cajón de sastre, para todo tipo de artrosis postraumáticas, con un abanico limitante (1 a 10 puntos) sin duda insuficiente, pues determinadas artrosis postraumáticas pueden ocasionar un menoscabo o discapacidad mucho más importante que el máximo considerado.

- Se presta a duplicar valoraciones por discapacidades únicas en lesiones combinadas.

- Existen importantes lagunas que obligan a tener que actuar de modo analógico, como la falta total de concreción en las secuelas por prótesis, con lesiones vasculares o nerviosas, tromboembolias, intolerancias, etc., relativamente frecuentes en la actualidad al prodigarse las indicaciones de las mismas, por lo que deberían ser objeto de mayor atención y especificación.

- No valora los trastornos ni la claudicación en la marcha, debiendo acudir a valoraciones por analogía (perjuicio estético dinámico).

Por todo ello, y dada la insuficiencia y la mala orientación del citado baremo, la valoración deberá basarse en un estudio de las alteraciones y de las discapacidades residuales, que debería comprender, de modo concreto:

- Valoración de la discapacidad para actividades físicas moderadas y/o intensas, especificando estas en la medida de lo posible (bipedestación, marcha, carrera, ascenso y descenso de escaleras, adopción de determinadas posturas, actividades deportivas, etc.).

- Evaluación del dolor permanente u ocasional, que requiera o no farmacoterapia.

- Limitación de la movilidad (flexoextensión y rotación), siempre tras la exploración comparativa de la rodilla contralateral.

- Posible inestabilidad de la articulación, determinando su grado, y valoración de la posibilidad

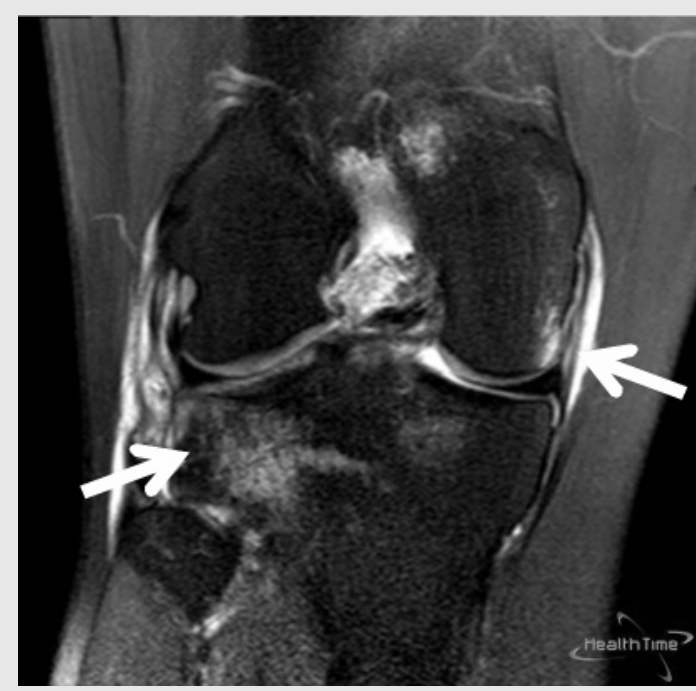

Figura 6.

T2 SPIR: engrosamiento $y$ edema del ligamento colateral interno ( $\mathrm{LCl})$ : esguince grado II.

Fractura trabecular subcondral en superficie articular posterior de platillo tibial externo.

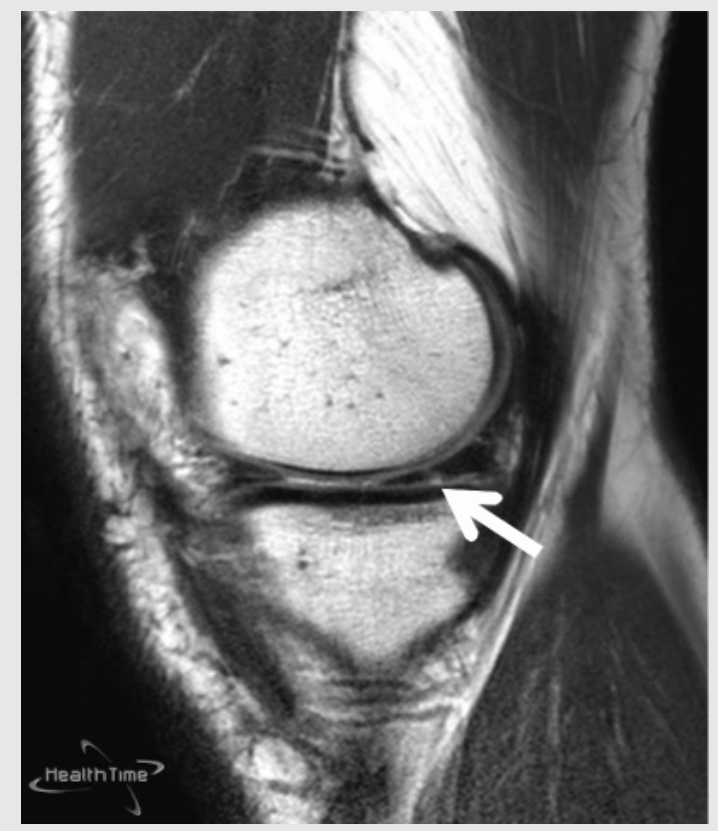

Figura 7.

T2 TSE SAG: fractura oblicua de cuerno posterior de menisco interno. de necesitar futuras actuaciones médicas o quirúrgicas.

- Concretar la existencia de lesiones específicas de la rodilla (óseas, meniscales, ligamentosas, rotulianas) y otras inespecíficas concomitantes (infecciosas, nerviosas, vasculares, tendinosas, cartilaginosas u óseas) que puedan requerir en un futuro tratamientos médicos o quirúrgicos.

- Considerar la existencia de estados patológicos previos de índole degenerativa, osteonecrótica u osteoporótica, que hayan podido verse 
agravados, y determinar su repercusión en la discapacidad existente.

- Valorar de manera específica y personalizada el material de osteosíntesis, especialmente de las prótesis.

- Evaluar las posibles deformidades o cicatrices existentes en relación al perjuicio estético, tanto estático como dinámico (cojeras).
- Realizar valoraciones analógicas cuando las necesidades del caso concreto así lo requieran.

Los autores declaran que el presente trabajo no ha sido subvencionado total o parcialmente por alguna entidad pública o privada y que no existe ningún tipo de conflicto de intereses.

\section{Bibliografía}

- Alonso Benavente A, López Alameda S, Rodrigo Verguizas, A. Exploración clínica y diagnóstica por la imagen de la rodilla. En: Manual del residente de COT. Madrid: SECOT; 2009. p. 339-45.

- García Prieto E, Alonso Moreno FJ, Vicario Espinosa C, Jiménez Pina I. Exploración de rodilla y hombro. Medicina de Familia-SEMERGEN. 2009;35(10):517-22.

- Hoppenfeld S. Exploración física de la rodilla. En: Exploración física de la columna vertebral y las extremidades. México DF: El Manual Moderno; 1999. p. 301-51.

- Jurado Bueno A, Medina Porqueres I. Rodilla. En: Manual de pruebas diagnósticas. Traumatología y ortopedia. $2^{\mathrm{a}}$ ed. Badalona: Paidotribo 2007; p. 209-68.

- Mélennec L, Doury P. Aparato locomotor. Extremidades inferiores. En: Valoración de las discapacidades y del daño corporal. Baremo internacional de invalideces. Barcelona: Masson; 2000. p. 205-22.
- Palomar M, Linares R. Anatomía de la articulación de la rodilla. En: Basas A, Fernández $C$, Martín JA, editores. Tratamiento fisioterápico de la rodilla. Madrid: McGraw-Hill; 2003. p. 1-15.

- Ryzewicz M, Peterson B, Siparsky PN, Bartz RL. The diagnosis of meniscus tears. The role of MRI and clinical examination. Clin Orthoped Relat Res. 2007;455:123-33.

- Texto refundido de la Ley sobre responsabilidad civil y seguro en la circulación de vehículos a motor. Real Decreto Legislativo 8/2004, de 29 de octubre. Boletín Oficial del Estado, núm. 267 (5-11-2004).

- Vázquez-Arce MI, et al. Valoración clínica e instrumental en la artrosis de rodilla. Rehabilitación. 2009;43(5):223-31.

- Yacut E, Bayar B, et al. Confiabilidad y validez de la escala visual analógica invertida (de derecha a izquierda) en dolores de diferente intensidad. The Pain Clinic. 2003;15(1):1-6. 